\title{
Investigation of Heat Transfer in a Microchannel with Same Heat Capacity Rate
}

\author{
Bin $\mathrm{Xu}^{1, *}$, Teck Neng Wong ${ }^{2}$, Nam-Trung Nguyen ${ }^{3}$ \\ ${ }^{1}$ Research Center of Fluid Machinery Engineering and Technology, Jiangsu University, \\ 301 Xuefu Road, Zhenjiang 212013 \\ ${ }^{2}$ School of Mechanical and Aerospace Engineering, Nanyang Technological \\ University, 50 Nanyang Avenue, Singapore 639798 \\ ${ }^{3}$ Queensland Micro- and Nanotechnology Centre, Griffith University, 170 Kessels \\ Road, Brisbane 4111, Australia
}

\begin{abstract}
In this paper, a new experimental setup was proposed to realize the constant-heat-flux boundary condition based on a counter flow microchannel heat exchanger with the same heat capacity rate of the hot and cold streams. This approach provides a constant fluid temperature gradient along the surfaces. An analytical two-dimensional model was developed to describe the heat transfer processes in the hot stream and the cold stream, respectively. In the experiments, DI-water was employed as the working fluid. Laser induced fluorescence (LIF) method was used to measure the fluid temperature field within the microchannel. Different combinations of flow rates were studied to investigate the heat transfer characteristics in the microchannel. The measured mean temperature distribution matched well with the proposed analytical model. A correlation for Nusselt number $(\mathrm{Nu})$ was proposed based on the experimental Reynolds number $(\mathrm{Re}<1)$ and Prandtl number $(\mathrm{Pr})$.
\end{abstract}

Key words: Heat transfer, Nusselt number, counter flow, microchannel

*Corresponding author. E-mail: norkistar@ujs.edu.cn 


\section{Introduction}

In the last decade, due to the rapid development of micro-electronics and biotechnology, micro heat exchangers (MHE) have attracted increasing attention from the research community [1]. Compared with conventional heat sinks and heat pipes, micro heat exchangers are proved to be effective in removing high heat fluxes which take advantage of the large surface-to-volume ratio and short transport path [2]. Therefore, there is a need for understanding fluid flow and heat transfer characteristics in a microchannel [3]. Followed by Tuckerman's [4] initial work on the fluid flow and heat transfer in a rectangular microchannel, many researchers have conducted related studies. Comprehensive review articles related to heat transfer characteristics of single-phase flow in microchannels have been presented [5, 6]. In the following section, selected literature related to the present study is highlighted.

The mode of flow in microchannels mostly remains under laminar flow regime due to small hydraulic diameter of the microchannel and eventually the heat transfer characteristic needs to be investigated. In the past few decades, experimental studies on the heat transfer characteristics in microchannels have been carried out by numerous researchers. However, the experimental studies on the heat transfer characteristics in microchannels pay attention to relatively high Reynolds number $(\operatorname{Re}>10)$. There have been very few works relating to relatively low Reynolds number $(\operatorname{Re}<10)$. Peng and Wang [7] investigated convective heat transfer characteristics for water flowing in rectangular microchannels with a width of $600 \mu \mathrm{m}$ and a height of $700 \mu \mathrm{m}$. The variation of $\mathrm{Nu}$ with Re was found to be unusual: Nu decreasing with increasing $\mathrm{Re}$ in laminar regime $(1000<\mathrm{Re}<3000)$. It was found that the experimental $\mathrm{Nu}$ for low values of Re was lower than the predictions of the Sieder-Tate correlation. Wang and Peng [8] conducted experimental investigation on liquid forced convection heat transfer through rectangular microchannels. Water was employed as the working fluid. The experimental $\mathrm{Nu}$ in the turbulent regime $(\mathrm{Re}>$ 3000) can be predicted by a modified Dittus-Boelter equation where the empirical 
constant coefficient was modified from 0.023 to 0.00805 . As a result, the experimental $\mathrm{Nu}$ was lower than the prediction of the conventional theory. Peng et al. [9] studied the effect of aspect ratio of microchannel on the $\mathrm{Nu}$ in both laminar and turbulent regimes. The $\mathrm{Nu}$ was correlated with microchannel dimensions. The $\mathrm{Nu}$ was found to be lower than the conventional values. Peng and Peterson [10-12] investigated the effect of Reynolds number (200 < Re $<1500$ ), the Prandtl number, the aspect ratio and dimensions on the heat transfer characteristics in the microchannel. The water property was temperature dependent. Cuta et al. [13] measured the $\mathrm{Nu}$ of a micro heat exchanger. The $\mathrm{Nu}$ was found to be proportional to $\operatorname{Re}(100<\operatorname{Re}<570)$, which was larger than the conventional values in laminar and turbulent regime. Ravigururajan et al. [14] conducted experiments on the laminar convective heat transfer through rectangular microchannels. The heat transfer coefficient was found to be three times higher than the conventional value. Tso and Mahulikar [15] investigated the effect of viscous dissipation by means of the Brinkman number $(\mathrm{Br})$ on the convective heat transfer characteristics in rectangular microchannels $(10<\operatorname{Re}<50)$. On the one hand, the Brinkman number was found to be too low (of the order of $10^{-8}$ ) to affect the water bulk temperature by means of the viscous dissipation (primary effect of Brinkman number). On the other hand, convective heat transfer in rectangular microchannels could be affected through the axial variation of the Brinkman number (secondary effect of Brinkman number), which results in the unusual behavior: $\mathrm{Nu}$ decreases with increasing Re in laminar regime. The author concluded that there did not have a fully developed region in microchannels due to the axial variation of Brinkman number. Rahman et al. $[16,17]$ measured experimental $\mathrm{Nu}$ in rectangular microchannels $(500<\operatorname{Re}<3500)$. The experimental $\mathrm{Nu}$ was found to be higher than the conventional correlations. The difference between experimental and conventional value was due to the surface roughness. Gao et al. [18] conducted experiment to study the variation of $\mathrm{Nu}$ along the axial direction in a rectangular microchannel $(\mathrm{Re}>200)$. The channel had a width of $25 \mathrm{~mm}$ and a height ranging from 0.1 to $1 \mathrm{~mm}$. The measured $\mathrm{Nu}$ agreed well with the conventional values when the channel height was larger than $0.4 \mathrm{~mm}$. On the other 
hand, the measured $\mathrm{Nu}$ was much higher than the conventional value when the channel height was less than $0.4 \mathrm{~mm}$. Wu and Cheng [19] investigated heat transfer in thirteen different trapezoidal microchannels. The Nu increased with surface roughness and $\operatorname{Re}(\operatorname{Re}<100)$. Lee and Garimella [20, 21] numerically investigated laminar convective heat transfer in the entrance region of rectangular cross-section microchannels which were subjected to circumferentially uniform wall temperature and axially uniform wall heat flux thermal boundary conditions $(\mathrm{Re}>300)$. Based on the numerical simulation results, a correlation was proposed to predict the local and average $\mathrm{Nu}$ which was a function of the dimensionless axial distance and channel aspect ratio. The proposed correlation matched well with some available experimental data. Zhigang et al. [22] conducted experiment to study convective heat transfer of water in micro-tubes. The experimental Nusselt number was found to be less than the predictions of the conventional laminar correlations at lower Reynolds number $(\operatorname{Re}>$ 1000). The effects of the variation of the thermo-physical properties with temperature and nonlinear temperature variation of the working fluid along the wall were found to be responsible for the discrepancy. Park and Punch [23] studied heat transfer in multiple microchannels with uniform flow distribution $(69<\mathrm{Re}<800)$. It was found that there were deviations between experimental and conventional $\mathrm{Nu}$. An empirical correlation for $\mathrm{Nu}$ was proposed as a function of Re, Pr and Br. Garcia-Hernando et al. [24] experimentally investigated fluid flow and heat transfer in a micro-heat exchanger $(\mathrm{Re}>10)$. The heat transfer characteristics were found to match well with the conventional theory expect for very low Re. Moharana et al. [25] studied axial conduction in single-phase simultaneously developing flow in a rectangular microchannel experimentally and numerically $(150<\operatorname{Re}<2000)$. No new physical phenomenon was observed based on these experimental results.

Based on the previous literature review, we can see that experimental investigation on fluid flow and heat transfer in microchannels mainly focus on the development of correlations for $\mathrm{Nu}$ as a function of $\mathrm{Re}, \mathrm{Pr}, \mathrm{Br}$ and microchannel dimensions. The limitation of the previous studies is that they all focus on the heat transfer 
characteristics where Reynolds number is larger than 10. Besides, the use of thermocouple to measure fluid temperature in the microchannel will affect fluid flow and heat transfer characteristics as the size of the thermocouple is on the same order as that of the microchannel. In this paper, fluid flow and heat transfer characteristics in a rectangular microchannel will be investigated theoretically and experimentally at very low Reynolds numbers ( $\operatorname{Re}<1)$. A new experimental setup is proposed to study heat transfer in a counter flow microchannel to provide the constant-heat-flux boundary condition. Two heat transfer zones (for the hot stream and the cold stream) are analyzed and investigated. An analytical model is proposed for the heat transfer process of the constant-heat-flux microchannel. Experiments are carried out to validate the theoretical model. Laser Induced Fluorescence (LIF) method is employed to measure the internal fluid temperature in the microchannel, which is based on the temperature-dependent fluorescence. The Nusselt number can be obtained by measuring the wall temperature and mean temperature of hot and cold streams. A correlation for the Nusselt number is subsequently developed to show the heat transfer characteristics in microchannels.

\section{Experiment}

\subsection{Materials and fabrications}

The experimental setup is shown in Figure 1(a). Polymethylmethacrylate (PMMA) is employed in the experiments due to the easy fabrication using a $\mathrm{CO}_{2}$ laser cutting system (Universal Laser Systems Inc., laser power of $25 \mathrm{~W}$, and a maximum beam speed of about $640 \mathrm{~mm} / \mathrm{s}$ ). The width of the microchannel was determined by the width of the drawn lines, where the depth of the microchannel was controlled by the power and the speed of the laser beam. The fabrication is based on the thermal bonding technique [26]. In this method, three PMMA plates $(50 \mathrm{~mm} \times 50 \mathrm{~mm})$ were bonded together to form a closed microchannel with inlets and outlets. The two PMMA parts are thermally bonded using a hot press (CARVER manual press 4386). The temperature of the hot plate was kept at $80{ }^{\circ} \mathrm{C}$ for 20 minutes, subsequently 
raised to $170{ }^{\circ} \mathrm{C}$ and then kept for another 20 minutes. Finally, the device was allowed to cool down to the room temperature. After thermal bonding process, a micro-heat exchanger with a cross section of $500 \mu \mathrm{m} \times 1000 \mu \mathrm{m}$ was fabricated.

\subsection{Chemical and experimental setup}

In this experiment, the working fluid is the De-ionized water (DI-water). The temperature dependent fluorescence Rhodamine $\mathrm{B}\left(\mathrm{C}_{28} \mathrm{H}_{31} \mathrm{~N}_{2} \mathrm{O}_{3} \mathrm{Cl}\right)$ was added into the DI-water at a concentration of $0.025 \mathrm{~g} / 100 \mathrm{ml}$. The relationship between fluorescence intensity (normalized by the intensity at $25{ }^{\circ} \mathrm{C}$ ) and temperature is shown in Figure 2 [27, 28]. Hot and cold Rhodamine B solutions are introduced into the counter flow microchannel by two identical syringe pump (Cole-Parmer, $74900-05,0.2 \mu \mathrm{l} / \mathrm{h}$ to $500 \mathrm{l} / \mathrm{h}$, accuracy of $0.5 \%$ ). A low voltage power supply (GW Model GPC- 30300) was used to supply voltage to a thermoelectric module (TEC) to heat up the Rhodamine B solution in the microchannel. A thermoelectric module is a solid-state method of heat transfer by means of different semiconductor materials, which is also commonly known as the Peltier effect. The voltage was supplied to enable heating/cooling in the microchannel. An interline transfer CCD camera (Sony ICX 084) attached to the microscope is employed to capture the fluorescent images.

\subsection{Experimental technique to realize constant-heat-flux boundary condition}

Based on the literature review, an electric resistance wire which is wrapped around the microchannels or a cartridge heater [29, 30] is employed to provide constant-heat-flux boundary condition. The main drawback of this method is that the heat loss to the surrounding environment, which will lead to non-uniform distribution of heat flux boundary condition. This paper attempts to propose a counter flow microchannel with the same heat capacity rates to realize a constant-heat-flux boundary condition. In this configuration, constant-heat-flux boundary condition will be obtained spontaneously due to the heat transfer between the hot and cold streams in the microchannel. The fabricated device used in the experiment is shown in Figure 1(b). The enlarged 
schematic illustration of the counter flow microchannel is shown in Figure 1(c). To accurately describe the formation of constant-heat-flux boundary condition in the counter flow microchannel, the heat transfer processes are divided into the upper fluid channel and the lower fluid channel. In the microchannel, a hot stream with a high temperature $T_{h, i}$ flows into the inlet arms of upper fluid channel (a-b-d-c), while a cold stream with a low temperature $T_{c, i}$ flows into the inlet arms of lower fluid channel (l-m-o-n). Subsequently, the hot stream flows in the horizontal part of upper fluid channel (A-B-D-C) from left to right, while the cold stream flows in the horizontal part of lower fluid channel (E-F-H-G) from right to left. During the counter flow process, heat transfer from the hot stream to the cold stream through the wall of the PMMA plate. Hence, the hot stream will be cooled to a lower temperature $T_{h, o}$ leaving the outlet arms of upper fluid channel (e-f-h-g), while the cold stream will be heated to a higher temperature $T_{c, o}$ leaving the outlet arms of lower fluid channel (h-i-k-j). The hot and cold streams experience little or no change in their velocities and elevations, and thus the kinetic and potential energy changes are negligible. For an ideal circumstance where the outer surface of the heat exchanger is perfectly insulated, there is no heat loss to the surrounding environment. Under these assumptions, the rate of heat transfer from the hot stream is equal to the rate of heat transfer to the cold one. That is,

$$
\mathcal{\&}=\left(n \& c_{p}\right)_{h}\left(T_{h, i}-T_{h, o}\right)
$$

and

$$
\mathcal{Q}=\left(n \& c_{p}\right)_{c}\left(T_{c, o}-T_{c, i}\right)
$$

where the subscript $h$ and $c$ are for hot and cold streams, respectively. $n k, c_{p}$ and $T$ are the mass flow rate, specific heats and temperature of streams. For the hot and cold streams with the same heat capacity rates $\left(n \& c_{p}\right)_{h}=\left(n \& c_{p}\right)_{c}$, the temperature difference between two fluids is a constant value respect to $x$. For the actual experimental setup, it is difficult to perfectly insulate the outer surface of the heat 
exchanger. In our experiment, two rectangular slots near the upper and lower fluid channels were cut to avoid heat loss. Based on this configuration, the percentage of heat loss is about $8 \%$.

\section{Analytical model}

An analytical model is developed to describe the heat transfer process as shown in Figure 3. Figure 3(a) and 3(b) illustrate a two-dimensional model for the heat transfer process of the hot stream. The microchannel has a width of $W$ and a length of $L$. The flow in the rectangular channel is assumed to have a constant velocity of $U$. A hot stream with high temperature $T_{h, i}$ flows into the microchannel, while with low temperature $T_{h, o}$ leaving the channel. At the bottom wall $\left(y^{*}=0\right)$, a constant-heat-flux boundary condition is specified due to the heat exchanges between the hot and cold streams with the same heat capacity rates. At the upper wall $\left(y^{*}=1\right)$, a constant-heat-flux boundary condition is specified due to the heat loss from upper wall of the hot stream to the ambient environment is negligible due to the existence of the rectangular slot.

In the analytical model, assumptions are made as follows,

1. Laminar, incompressible, Newtonian fluid, steady flow is assumed;

2. Hot and cold streams have the same property; viscosity and thermal diffusivity are independent of temperature;

3. Convection is assumed to occur in one direction, axially along the microchannel;

4. Decoupled convection and diffusion processes.

The governing equation can be formulated in the dimensional form as follow:

$$
\alpha\left(\frac{\partial^{2} T}{\partial x^{2}}+\frac{\partial^{2} T}{\partial y^{2}}\right)=U \frac{\partial T}{\partial x}
$$

where $\alpha$ is the thermal diffusivity of the fluid $\left(\alpha=k /\left(\rho c_{p}\right)\right)$. By introducing the dimensionless coordinate system $x^{*}=x / W, y^{*}=y / W$, the normalized temperature 
$\Theta=\frac{T-T_{h, o}}{T_{h, i}-T_{h, o}}$ and the Peclet number,

$$
P e=\frac{U W}{\alpha}
$$

Energy equation (3) can be formulated in dimensionless form as:

$$
\frac{\partial^{2} \Theta}{\partial x^{* 2}}+\frac{\partial^{2} \Theta}{\partial y^{* 2}}=P e \frac{\partial \Theta}{\partial x^{*}}
$$

The inlet and outlet boundary conditions for Eq. (5) are:

$$
\left.\Theta\right|_{\left(x^{*}=0\right)}=1,\left.\Theta\right|_{\left(x^{*}=1\right)}=0
$$

The constant-heat-flux boundary condition is applied to the upper and bottom walls:

$$
\left.\frac{\partial \Theta}{\partial y^{*}}\right|_{y^{*}=0}=E_{2} W /\left(T_{h, i}-T_{h, o}\right),\left.\frac{\partial \Theta}{\partial y^{*}}\right|_{y^{*}=1}=E_{1} W /\left(T_{h, i}-T_{h, o}\right)
$$

Using the Finite Fourier Transform (FFT) method [31], and applying corresponding boundary conditions Eqs. (6)-(7), the dimensionless temperature distribution in the upper region of microchannel for the hot stream can be obtained

$$
\begin{aligned}
\Theta\left(x^{*}, y^{*}\right)= & A_{1}+A_{2} \exp (P e * x)-\left(E_{2}-E_{1}\right) * x / P e \\
& +\sqrt{2} \cos (n \pi y) \sum_{n=1}^{\infty} B_{1} \exp \left(\lambda_{1} * x\right)+B_{2} \exp \left(\lambda_{2} * x\right)-A
\end{aligned}
$$

where

$$
\begin{gathered}
A=\frac{\sqrt{2}\left[E_{2}-(-1)^{n} E_{1}\right]}{(n \pi)^{2}} \\
A_{2}=\left(1-\frac{E_{2}-E_{1}}{P e} L\right) /(1-\exp (P e * L)), \quad A_{1}=1-A_{2} \\
\lambda_{1,2}=\left(P e \pm \sqrt{P e^{2}+4(n \pi)^{2}}\right) / 2 \\
B_{2}=\frac{A\left(1-\exp \left(\lambda_{1} * L\right)\right)}{\exp \left(\lambda_{2} * L\right)-\exp \left(\lambda_{1} * L\right)}, \quad B_{1}=A-B_{2}
\end{gathered}
$$

A two-dimensional model of fluid flow in the lower region of counter flow microchannel is depicted in Figure 3(c) and 3(d). A cold stream with low temperature $T_{c, i}$ flows into the of the counter flow microchannel, while with high temperature 
$T_{c, o}$ leaving the channel. Similarly, the solution is,

$$
\begin{aligned}
\Theta\left(x^{*}, y^{*}\right)= & C_{1}+C_{2} \exp (P e * x)-\left(E_{3}-E_{4}\right) * x / P e \\
& +\sqrt{2} \cos (n \pi y) \sum_{n=1}^{\infty} D_{1} \exp \left(\lambda_{1} * x\right)+D_{2} \exp \left(\lambda_{2} * x\right)-B
\end{aligned}
$$

where

$$
\begin{gathered}
B=\frac{\sqrt{2}\left[E_{3}-(-1)^{n} E_{4}\right]}{(n \pi)^{2}} \\
C_{2}=\left(1+\frac{E_{3}-E_{4}}{P e} L\right) /(\exp (P e * L)-1), \quad C_{1}=-C_{2} \\
D_{2}=\frac{B\left(1-\exp \left(\lambda_{1} * L\right)\right)}{\exp \left(\lambda_{2} * L\right)-\exp \left(\lambda_{1} * L\right)}, \quad D_{1}=B-D_{2}
\end{gathered}
$$

where the temperature gradient $E_{1}, E_{2}, E_{3}$ and $E_{4}$ are determined from the measured surface temperature along $\mathrm{AB}, \mathrm{CD}, \mathrm{EF}$ and $\mathrm{GH}$ respectively. The measured temperature gradient along surface CD and EF is shown in Figure 4(d).

\section{Data analysis}

\subsection{Thermophysical property of water}

Properties of water are allowed to vary with temperature. The functional relations between density, specific heat, thermal conductivity and viscosity with temperature are described as follows [32],

$$
\begin{gathered}
\rho=\frac{a_{0}+a_{1} T+a_{2} T^{2}+a_{3} T^{3}+a_{4} T^{4}+a_{5} T^{5}}{1+a_{6} T} \\
c_{p}=b_{0}+b_{1} T+b_{2} T^{2}+b_{3} T^{3} \\
k=c_{0}+c_{1} T+c_{2} T^{2} \\
\mu=d_{0} 10^{d_{1} /\left(T-d_{2}\right)}
\end{gathered}
$$

In the above equations, thermal conductivity $k$ is in $\mathrm{W} / \mathrm{m} \cdot \mathrm{K}$, viscosity is in $\mathrm{Pa} \cdot \mathrm{s}$, specific heat $c_{p}$ is in $\mathrm{J} / \mathrm{kg} \cdot \mathrm{K}$ and temperature $T$ is in $\mathrm{K}$ except for Eq.(17) which is ${ }^{\circ} \mathrm{C}$ respectively. The various constants are listed in Table 1. 


\begin{tabular}{ccccc}
\hline$x$ & $a_{x}$ & $b_{x}$ & $c_{x}$ & $d_{x}$ \\
\hline 0 & 999.8396 & 8958.9 & -0.58166 & $2.414 \times 10^{-5}$ \\
1 & 18.22494 & -40.535 & $6.3555 \times 10^{-3}$ & 247.8 \\
2 & $-7.92221 \times 10^{-3}$ & 0.11243 & $-7.9643 \times 10^{-6}$ & 140.0 \\
3 & $-5.54485 \times 10^{-5}$ & $-1.0138 \times 10^{-4}$ & & \\
4 & $1.49756 \times 10^{-7}$ & & & \\
5 & $-3.933 \times 10^{-10}$ & & & \\
6 & $1.81597 \times 10^{-2}$ & & & \\
& & & \\
\end{tabular}

Table 1. Various constants related to the thermophysical property of water

\subsection{Local Nusselt number}

For thermal analysis [33, 34], the parameters used to analyze heat transfer with variable properties are determined by the mean temperature across the microchannel and the hot surface (CD, Fig. 1(c)) and the cold surface (EF, Fig. 1(c)). The parameters are summarized as:

The hydraulic diameter, $D_{h}$

$$
D_{h}=\frac{2 W H}{W+H}
$$

The Reynolds number, Re

$$
\operatorname{Re}=\frac{\rho_{l} U D_{h}}{\mu}
$$

The local convective heat transfer coefficient and the corresponding local Nusselt number along the microchannel were obtained from the Newton's law of cooling,

$$
\begin{gathered}
h=\frac{k_{f} \partial T / \partial y}{T_{m}-T_{s}} \\
N u=\frac{h D_{h}}{k_{l}}
\end{gathered}
$$

where $\partial T / \partial y$ is the measured fluid temperature gradient along CD and EF. The 
Brinkman number, $\mathrm{Br}$

$$
B r=\frac{\mu_{l} u_{m}^{2}}{k_{f} \partial T / \partial y * D_{h}}
$$

The Prandtl number, Pr

$$
\operatorname{Pr}=\frac{\mu_{l} c_{p}}{k_{l}}
$$

Where $W, H, \rho_{l}, U, \mu, k_{s}, c_{p}$ and $k_{l}$ are the microchannel width, height, fluid density, fluid velocity, fluid viscosity, PMMA wall thermal conductivity, fluid heat capacity and fluid thermal conductivity respectively.

\section{Results and discussion}

\subsection{Experimental results and discussion}

Figure 4(a)-4(b) shows the measured temperature profiles for hot and cold streams of which flowrate was maintained at $1000 \mu \mathrm{l} / \mathrm{h}$. Hot and cold streams with the same heat capacity rates flow oppositely into the respective inlet arms. The formation of temperature contours was observed, indicating that heat transfer between hot and cold streams occurred in the counter flow microchannel. Due to the heat transfer from hot stream to cold stream, the hot stream temperature decreases from $41^{\circ} \mathrm{C}$ to $36^{\circ} \mathrm{C}$, meanwhile the cold stream increases from $32.5^{\circ} \mathrm{C}$ to $36.5^{\circ} \mathrm{C}$. The resulting mean temperature distribution for the hot and cold stream and the surface temperature are shown in Figure 4(e). As demonstrated, a constant temperature gradient is maintained at the hot PMMA wall (CD) and the cold PMMA wall (EF) which indicate that a constant heat flux boundary condition was obtained in the experiment. The experimental temperature gradient is shown in Figure 4(f). A straight fitting line is placed to show an almost constant temperature gradient obtained in experiment. Figure 4(c)-4(d) shows the measured temperature profiles for hot and cold streams of which flowrate was maintained at $1750 \mu \mathrm{l} / \mathrm{h}$. Due to the heat transfer from the hot stream to the cold stream, the hot stream temperature decreases from $45^{\circ} \mathrm{C}$ to $40^{\circ} \mathrm{C}$, 
meanwhile the cold stream increases from $32{ }^{\circ} \mathrm{C}$ to $36{ }^{\circ} \mathrm{C}$. A uniform constant-heat-flux along the CD and EF was also observed as indicated by the circle.

The variation of the experimental local $\mathrm{Nu}$ with the channel length for the hot stream and cold stream is depicted in Figures 5(a)-5(b). The decrease of local Nu with the increase of channel length represents the existence of the entrance region, which is important for fluid flow and heat transfer in the counter flow microchannel. The experimental local $\mathrm{Nu}$ obtained in the microchannel can be correlated by the following correlation for the Reynolds number which is in the order of 1 (i.e. $\operatorname{Re}<1$ ),

$$
N u=1.0+\frac{1.6279 \times \operatorname{Re}^{1.4909} \operatorname{Pr}^{0.8678}\left(\frac{\mu_{m}}{\mu_{w}}\right)^{26.1441}\left(\frac{x}{L}\right)^{-0.0186}}{1.1523+0.0054 \times \operatorname{Re}^{-3.4244} \operatorname{Pr}^{1.9471}\left(\frac{\mu_{m}}{\mu_{w}}\right)^{-27.4877}\left(\frac{x}{L}\right)^{1.4529}}
$$

The correlation obtained in this study indicates that the $\mathrm{Nu}$ for fluid flow and heat transfer in rectangular microchannels is crucially dependent on the viscosity ratio ( $\left.\mu_{f} / \mu_{w}\right)$ and is moderately dependent on the Re and Pr. For Re $<1$, compared with other dimensionless number, $\mathrm{Br}$ is too low (of the order of $10^{-11}$ ) to influence the convective heat transfer characteristics in the microchannel. Therefore, $\mathrm{Br}$ is not included in the correlation. The comparison between the correlated $\mathrm{Nu}$ and experimental $\mathrm{Nu}$ are presented in terms of Root Mean Square error (RMS), which is defined as follows,

$$
\text { RMS error }=\left[\frac{1}{N} \sum_{1}^{N}\left(\frac{W_{\text {pred }}-W_{\text {exp }}}{W_{\exp }}\right)^{2}\right]^{1 / 2}
$$

where $W$ is the quantity being assessed such as the Nu. The RMS error gives the arithmetic mean absolute error of the data. Estimation of the RMS error in the experimental results is 0.02 . From the error analysis, we can conclude that there is small discrepancy between the correlated and experimental $\mathrm{Nu}$, which can be perceived as accurate. Therefore, the correlated formula can be used to predict the $\mathrm{Nu}$ in the case of low Re. The correlated $\mathrm{Nu}$ versus the experimental $\mathrm{Nu}$ is plotted in 
Figure 5(c). The Nu versus the Re at three different sections $(x / L=0.01, \quad x / L=0.5$ and $x / L=1)$ is shown in Figure $5(d)(\operatorname{Pr}=4)$. From this figure, we can see that the Nu increases as the Re increases. Besides, near the channel entrance ( $x / L=0.01$ ), the $\mathrm{Nu}$ increases immediately as Re increase. In the middle section of micro-heat exchanger $(x / L=0.5)$, the $\mathrm{Nu}$ will first experience a slow increase and then raises with the increase of Re. At the channel outlet $(x / L=1)$, the Nu will undergo a slower increase compared with the middle section. We can conclude that the closer to the entrance, the more sensitive of the variation of $\mathrm{Nu}$ versus Re.

\subsection{Comparison between analytical results and experimental results}

Analytical temperature distributions for the hot and cold streams are shown in Fig. 6(a)-6(b). Compared with the measured temperature profiles (Figs. 4(a)-4(b)), the analytical results agrees well with the experimental results, which demonstrate the usability of the boundary conditions setting for the heat transfer processes in the counter flow microchannel for the hot stream and cold stream respectively. The mean temperature distribution along the channel length is shown in Figure 6(c). As the channel length increases, the temperature difference between the hot stream and cold streams is almost a constant value due to the same heat capacity rate of the fluids.

\section{Conclusions}

In this paper, heat transfer in a microchannel with axially uniform wall constant-heat-flux is investigated theoretically and experimentally. With the help of the Laser-Induced Fluorescence (LIF) method, DI-water with temperature-dependent fluorescent Rhodamine $\mathrm{B}$ is employed to show the temperature field in the microchannel. The proposed method ensures a constant fluid temperature gradient along the wall. The measured mean temperature distributions match well with the analytical model. Based on the measured temperature field, local Reynolds number (Re), Prandtl number (Pr), and Nusselt number $(\mathrm{Nu})$ can be obtained. A correlation is developed. The convective heat transfer in microchannels was found to be 
significantly dependent on the viscosity ratio $\left(\mu_{f} / \mu_{w}\right)$. The Nusselt number was found to increase with the Reynolds number and the closer distance to the entrance, the more sensitive of the variation of Nusselt number versus Reynolds number.

Conflicts of Interest: On behalf of all authors, the corresponding author states that there is no conflict of interest. 


\section{References}

1. Chein, R. and Y. Chen, Performances of thermoelectric cooler integrated with microchannel heat sinks. International Journal of Refrigeration, 2005. 28(6): p. 828-839.

2. Zhao, Y., G. Chen, and Q. Yuan, Liquid-liquid two-phase mass transfer in the T-junction microchannels. AIChE Journal, 2007. 53(12): p. 3042-3053.

3. Nguyen, N.T., D. Bochnia, R. Kiehnscherf, and W. Dötzel, Investigation of forced convection in microfluid systems. Sensors and Actuators, A: Physical, 1996. 55(1): p. 49-55.

4. Tuckerman, D.B. and R.F.W. Pease, HIGH-PERFORMANCE HEAT SINKING FOR VLSI. Electron device letters, 1981. EDL-2(5): p. 126-129.

5. Obot, N.T., Toward a better understanding of friction and heat/mass transfer in microchannels - A literature review. Microscale Thermophysical Engineering, 2002. 6(3): p. 155-173.

6. Morini, G.L., Single-phase convective heat transfer in microchannels: A review of experimental results. International Journal of Thermal Sciences, 2004. 43(7): p. 631-651.

7. Peng, X.F. and B.X. Wang, Forced convection and flow boiling heat transfer for liquid flowing through microchannels. International Journal of Heat and Mass Transfer, 1993. 36(14): p. 3421-3427.

8. Wang, B.X. and X.F. Peng, Experimental investigation on liquid forced-convection heat transfer through microchannels. International Journal of Heat and Mass Transfer, 1994. 37(SUPPL. 1): p. 73-82.

9. Peng, X.F., G.P. Peterson, and B.X. Wang, Frictional flow characteristics of water flowing through rectangular microchannels. Experimental Heat Transfer, 1994. 7(4): p. 249-264.

10. Peng, X.F. and G.P. Peterson, Convective heat transfer and flow friction for water flow in microchannel structures. International Journal of Heat and Mass Transfer, 1996. 39(12): p. 2599-2608.

11. Peng, X.F. and G.P. Peterson, The effect of thermofluid and geometrical parameters on convection of liquids through rectangular microchannels. International Journal of Heat and Mass Transfer, 1995. 38(4): p. 755-758.

12. Peng, X.F., B.X. Wang, G.P. Peterson, and H.B. Ma, Experimental investigation of heat transfer in flat plates with rectangular microchannels. International Journal of Heat and Mass Transfer, 1995. 38(1): p. 127-137.

13. Cuta, J.M., C.E. McDonald, and A. Shekarriz, Forced convection heat transfer in parallel channel array microchannel heat exchanger. American Society of Mechanical Engineers, Heat Transfer Division, (Publication) HTD, 1996. 338: p. 17-23.

14. Ravigururajan, T.S., J. Cuta, C.E. McDonald, and M.K. Drost, Single-phase flow thermal performance characteristics of a parallel micro-channel heat exchanger. American Society of Mechanical Engineers, Heat Transfer Division, (Publication) HTD, 1996. 329: p. 157-161.

15. Tso, C.P. and S.P. Mahulikar, Experimental verification of the role of Brinkman number in microchannels using local parameters. International Journal of Heat and Mass Transfer, 2000. 43(10): p. 1837-1849.

16. Rahman, M.M., Measurements of heat transfer in microchannel heat sinks. International Communications in Heat and Mass Transfer, 2000. 27(4): p. 495-506.

17. Kandlikar, S.G., S. Joshi, and S. Tian. Effect of channel roughness on heat transfer and fluid 
flow characteristics at low reynolds numbers in small diameter tubes. in Proceedings of the National Heat Transfer Conference. 2001.

18. Gao, P., S. Le Person, and M. Favre-Marinet, Scale effects on hydrodynamics and heat transfer in two-dimensional mini and microchannels. International Journal of Thermal Sciences, 2002. 41(11): p. 1017-1027.

19. Wu, H.Y. and P. Cheng, An experimental study of convective heat transfer in silicon microchannels with different surface conditions. International Journal of Heat and Mass Transfer, 2003. 46(14): p. 2547-2556.

20. Lee, P.S., S.V. Garimella, and D. Liu, Investigation of heat transfer in rectangular microchannels. International Journal of Heat and Mass Transfer, 2005. 48(9): p. 1688-1704.

21. Lee, P.S. and S.V. Garimella, Thermally developing flow and heat transfer in rectangular microchannels of different aspect ratios. International Journal of Heat and Mass Transfer, 2006. 49(17-18): p. 3060-3067.

22. Zhigang, L., G. Ning, and M. Takei, An experimental investigation of single-phase heat transfer in $0.045 \mathrm{~mm}$ to $0.141 \mathrm{~mm}$ microtubes. Nanoscale and Microscale Thermophysical Engineering, 2007. 11(3-4): p. 333-349.

23. Park, H.S. and J. Punch, Friction factor and heat transfer in multiple microchannels with uniform flow distribution. International Journal of Heat and Mass Transfer, 2008. 51(17-18): p. 4535-4543.

24. García-Hernando, N., A. Acosta-Iborra, U. Ruiz-Rivas, and M. Izquierdo, Experimental investigation of fluid flow and heat transfer in a single-phase liquid flow micro-heat exchanger. International Journal of Heat and Mass Transfer, 2009. 52(23-24): p. 5433-5446.

25. Moharana, M.K., G. Agarwal, and S. Khandekar, Axial conduction in single-phase simultaneously developing flow in a rectangular mini-channel array. International Journal of Thermal Sciences, 2011. 50(6): p. 1001-1012.

26. Sun, Y., Y.C. Kwok, and N.T. Nguyen, Low-pressure, high-temperature thermal bonding of polymeric microfluidic devices and their applications for electrophoretic separation. Journal of Micromechanics and Microengineering, 2006. 16(8): p. 1681-1688.

27. Xu, B., T.N. Wong, N.T. Nguyen, Z. Che, and J.C.K. Chai, Thermal mixing of two miscible fluids in a T-shaped microchannel. Biomicrofluidics, 2010. 4(4): p. 1-13.

28. Xu, B., T.N. Wong, and N.T. Nguyen, Experimental and numerical investigation of thermal chaotic mixing in a T-shaped microchannel. Heat and Mass Transfer/Waerme- und Stoffuebertragung, 2011: p. 1-9.

29. San Andŕs, L., K. Ryu, and T.H. Kim, Thermal management and rotordynamic performance of a hot rotor-gas foil bearings system-part I: Measurements. Journal of Engineering for Gas Turbines and Power, 2011. 133(6).

30. Williamsen, M.S., S.K. Ray, Y. Zou, J.A. Dudek, S. Sen, M. Bissen, L. Kretsch, V.R. Palkar, M.F. Onellion, and P. Guptasarma, Ultrahigh vacuum sample mount for $x$-ray photoelectron spectroscopy up to very high temperature (150-1400 K). Journal of Vacuum Science and Technology A: Vacuum, Surfaces and Films, 2011. 29(3).

31. DEEN.W.M, Analysis of Transport Phenomena. 1998, New York: Oxford University Press.

32. Incropera, F.P., Liquid Cooling of Electronic Devices by Single-Phase Convection. 1999: John Wiley \& Sons, Inc.

33. HONG Feng, Y.J., and ZHOU Banglun, Numerical analysis of cavitating flow characteristics 
in impeller of residual heat removal pump. Journal of Drainage and Irrigation Machinery Engineering, 2016. 34(3): p. 185-190.

34. ZHANG Keyu, Y.J., SUN Wenting and SI Qiaorui, Internal flow characteristics of residual heat removal pump during different starting periods. Journal of Drainage and Irrigation Machinery Engineering, 2017. 35(3): p. 192-199. 


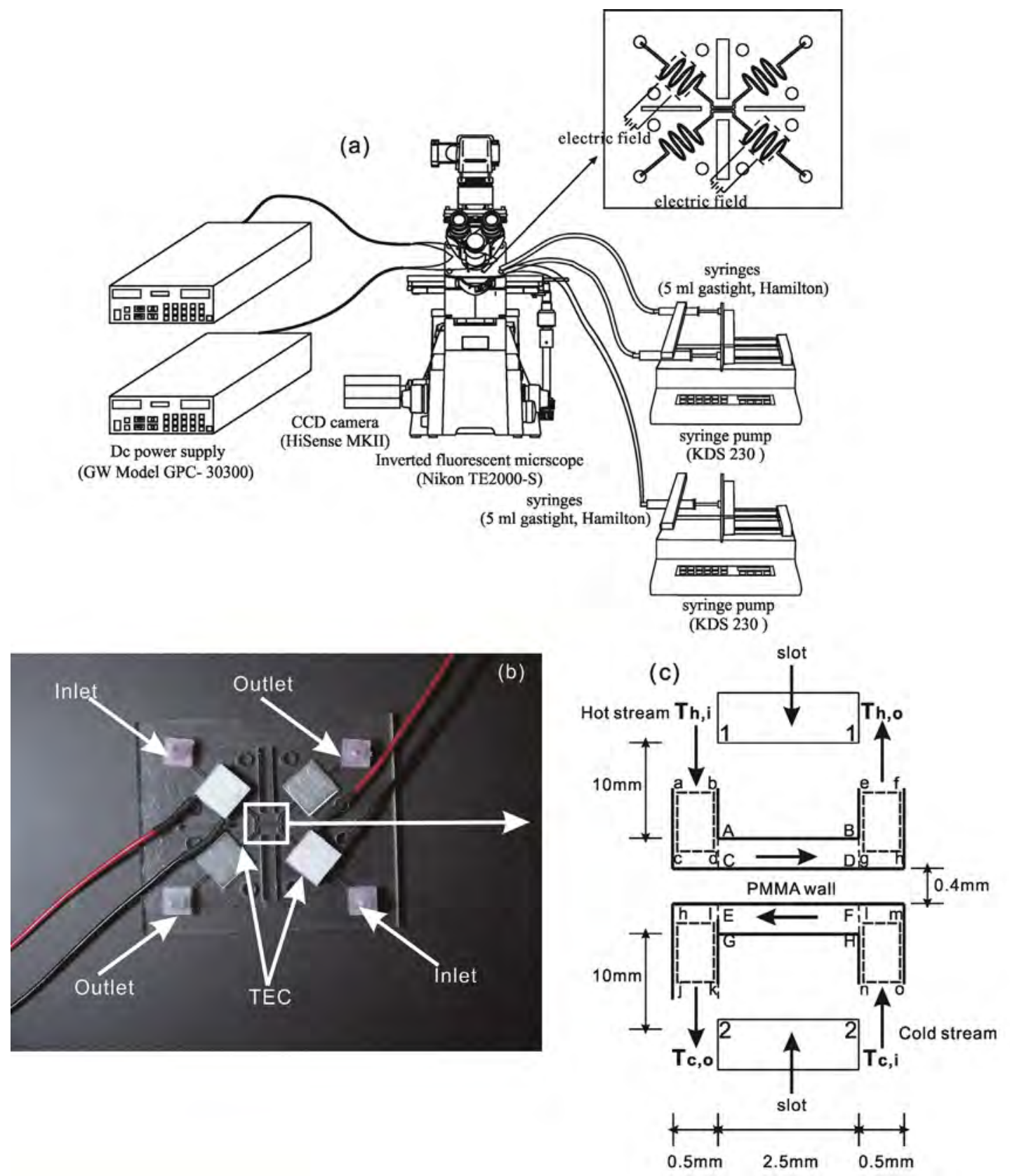

Figure 1. Experimental setup: (a) Schematic illustration, (b) The fabricated device used in the experiment, (c) Enlarged illustration of the focus area captured by CCD camera for counter flow microchannel 

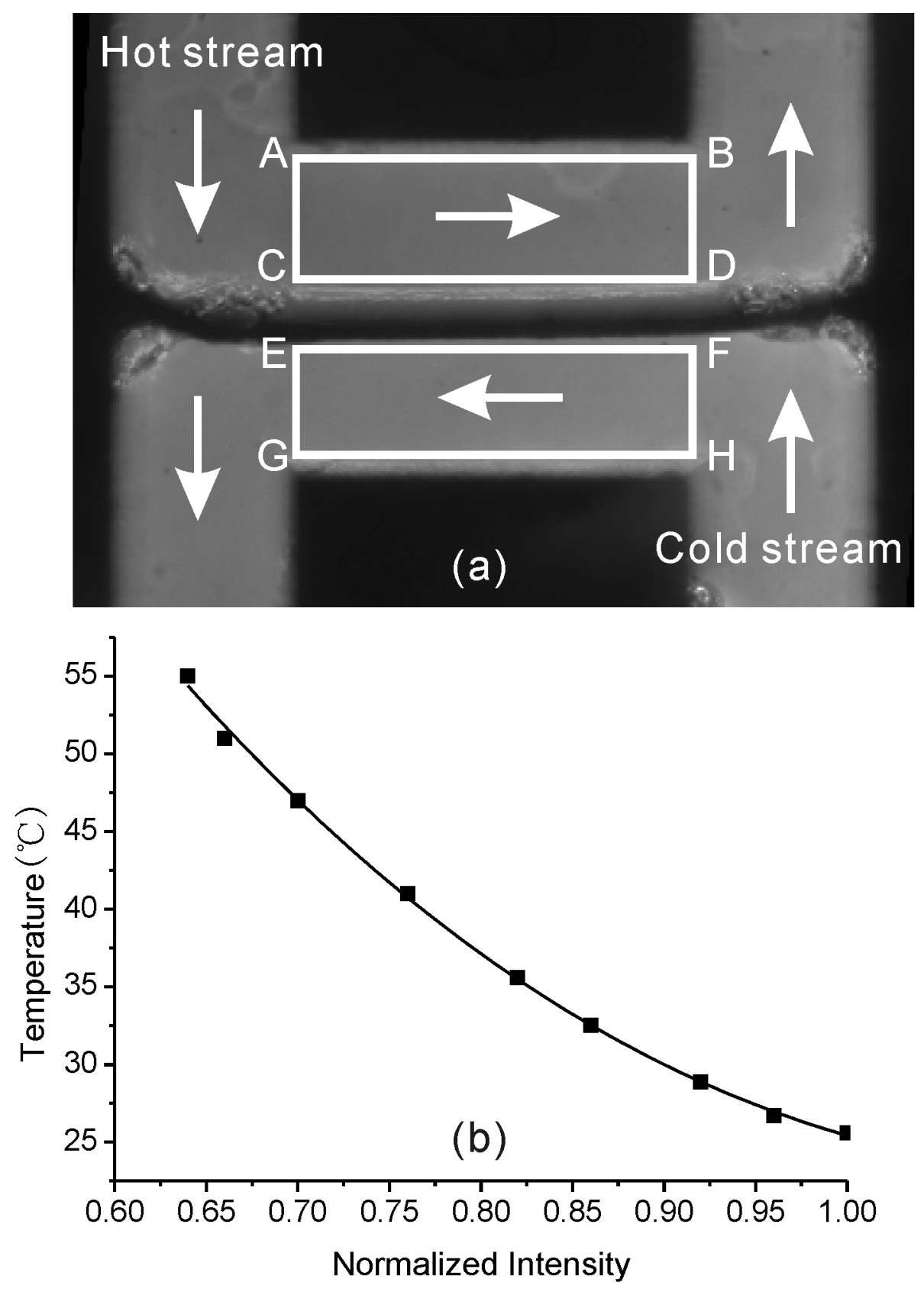

Figure 2. (a) A typical gray scale image for the counter flow microchannel heat exchanger, (b) Normalized intensity as a function of temperature 


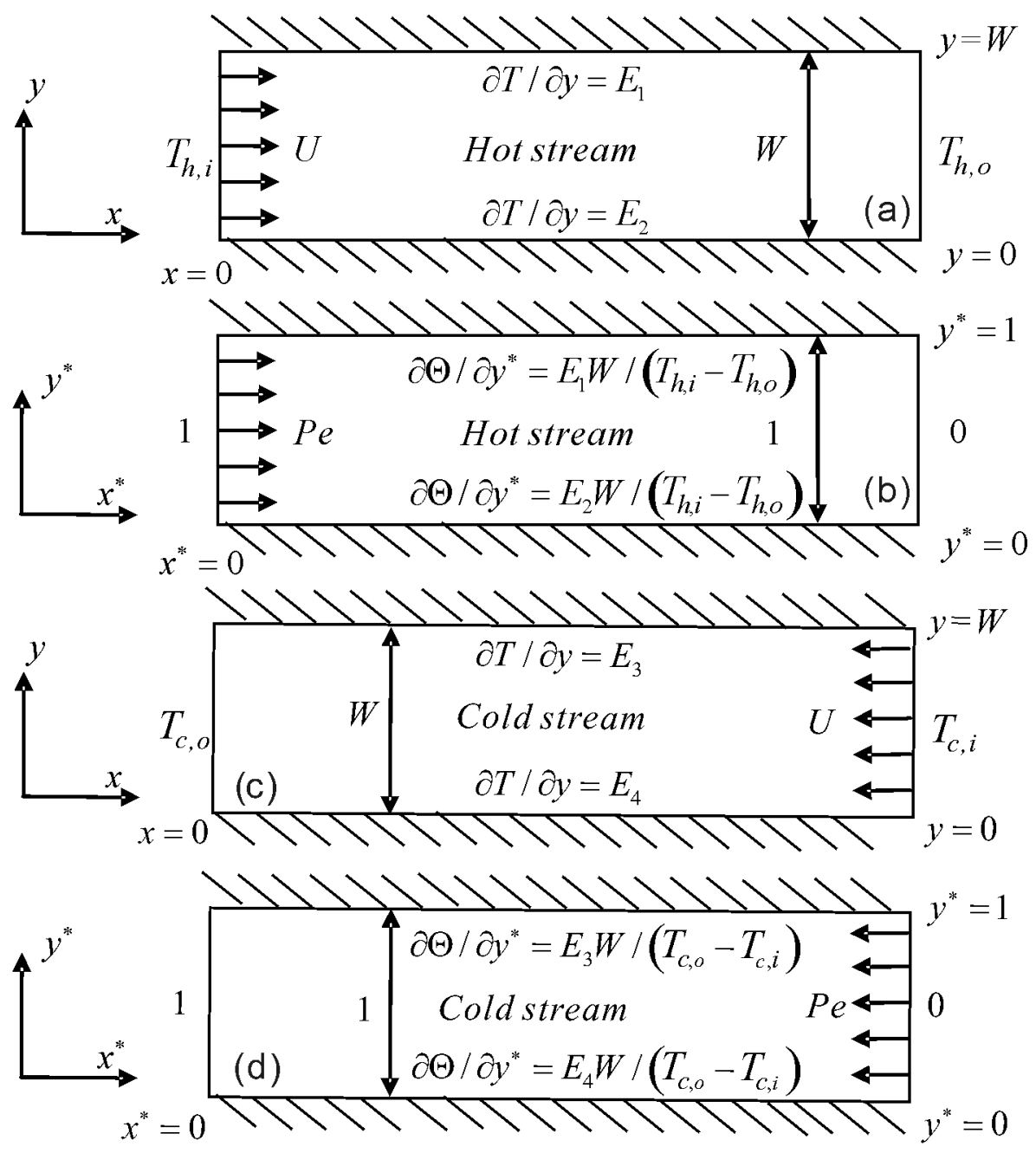

Figure 3. Two-dimensional model of heat transfer in the counter flow heat exchanger:

(a) the actual model (hot stream), (b) the dimensionless model (hot stream),

(c) the actual model (cold stream), (d) the dimensionless model (cold stream) 

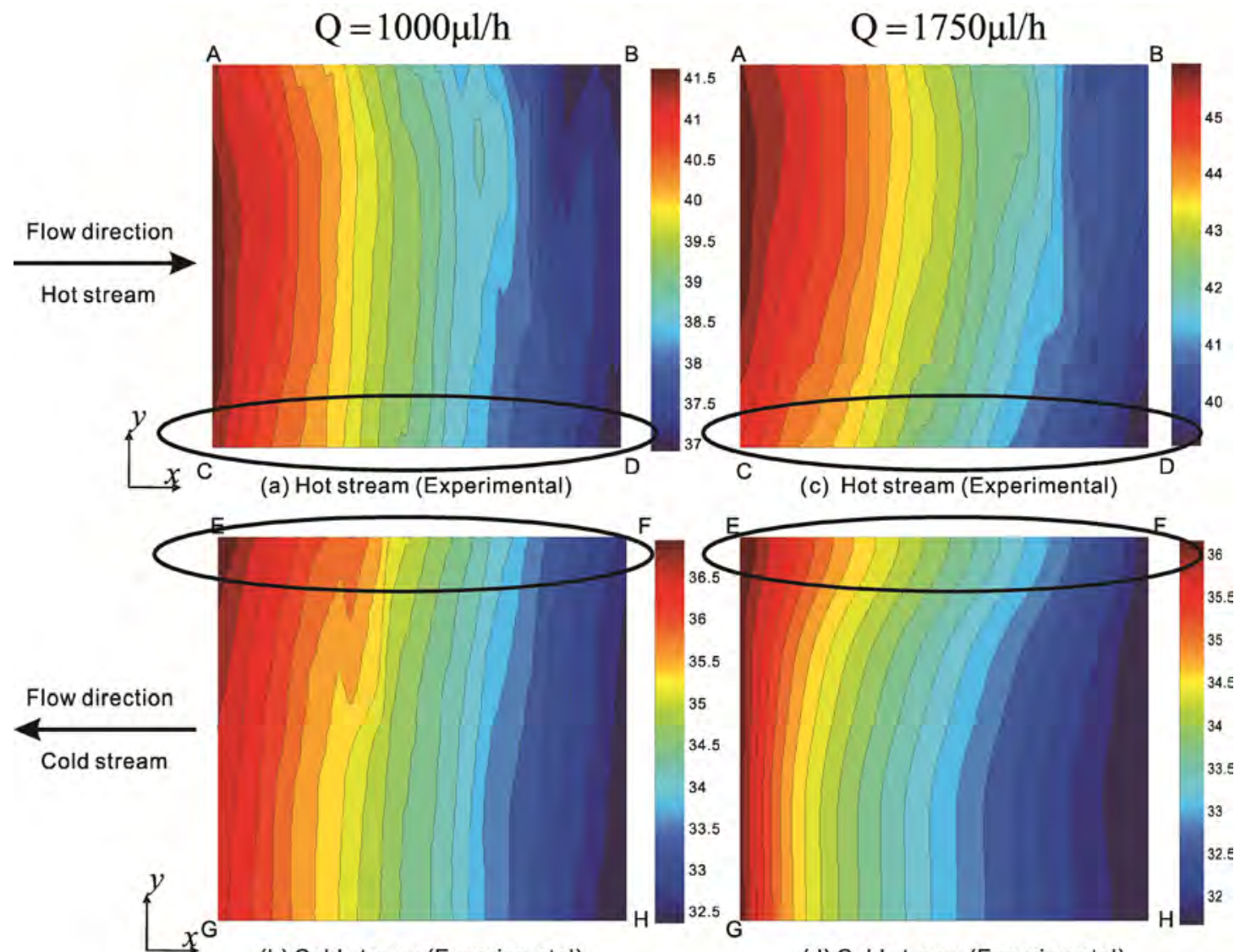

(b) Cold stream (Experimental)

(d) Cold stream (Experimental)
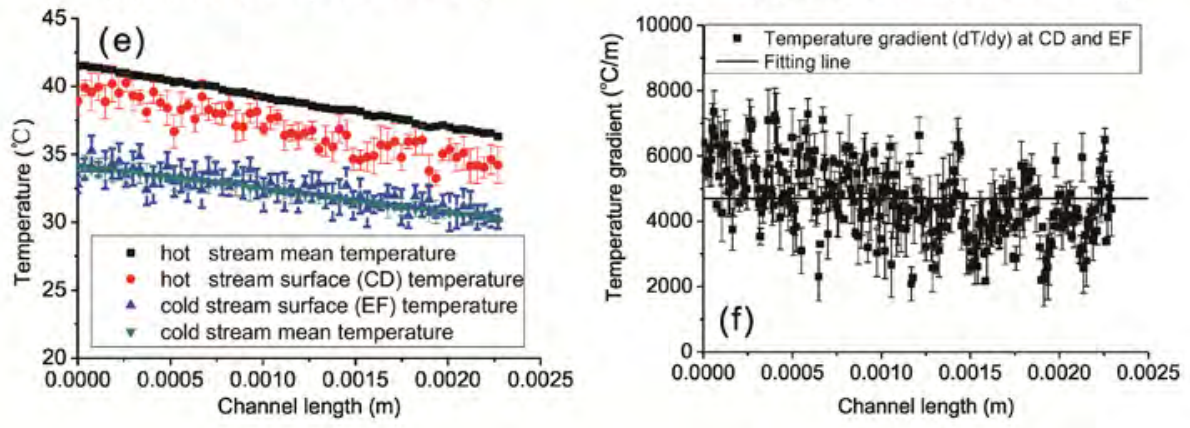

Figure 4. Experimental temperature distribution in microchannel

(a) temperature contours of hot stream ( $\mathbb{l}^{\&}=1000 \mu \mathrm{l} / \mathrm{h}$ ), (b) temperature contours of cold stream ( $\mathscr{L}^{\&}=1000 \mu \mathrm{l} / \mathrm{h}$ ), (c) temperature contours of hot stream ( $I^{\&}=1750 \mu \mathrm{l} / \mathrm{h}$ ), (d) temperature contours of cold stream ( $\&=1750 \mu \mathrm{l} / \mathrm{h})$,

(e) temperature profiles of mean and surface temperature ( $l^{\&}=1000 \mu \mathrm{l} / \mathrm{h}$ ), (f) temperature gradient along surface CD and EF ( $\&=1000 \mu \mathrm{l} / \mathrm{h}$ ) 

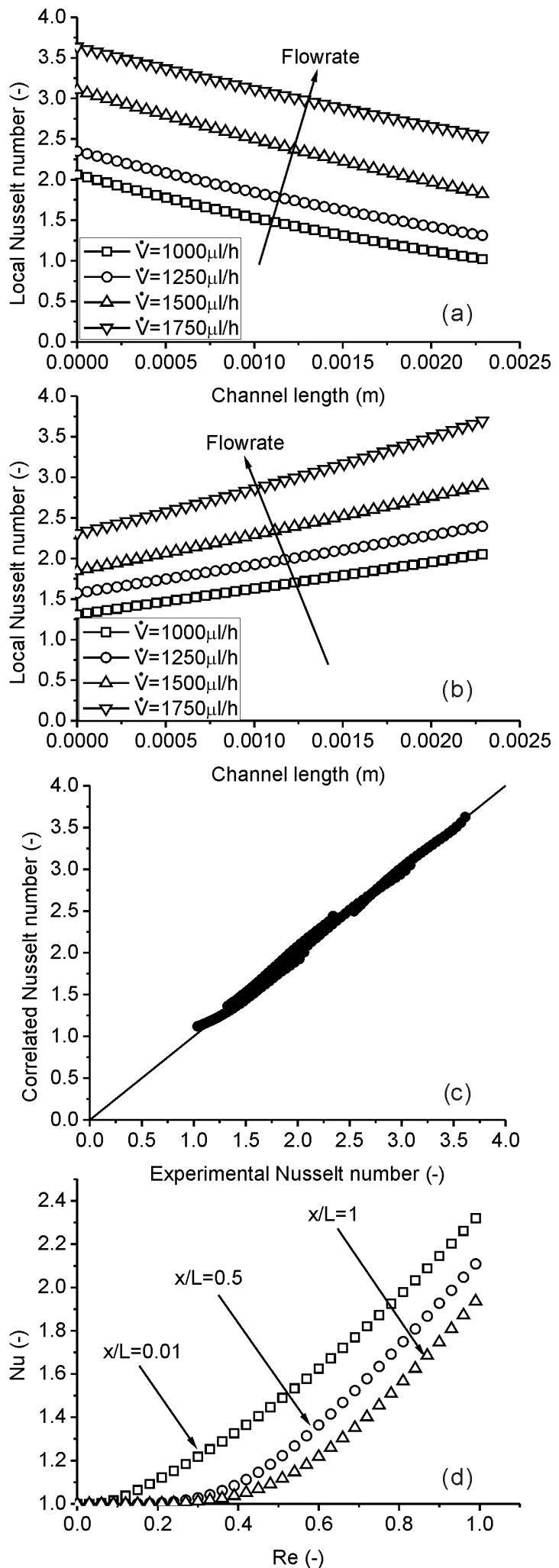

Figure 5. Experimental Nusselt number: (a) local Nu versus channel length for the hot stream, (b) local Nu versus channel length for the cold stream, (c) Experimental Nu versus Empirical Nu, (d) variation of Nu versus Re at different sections 

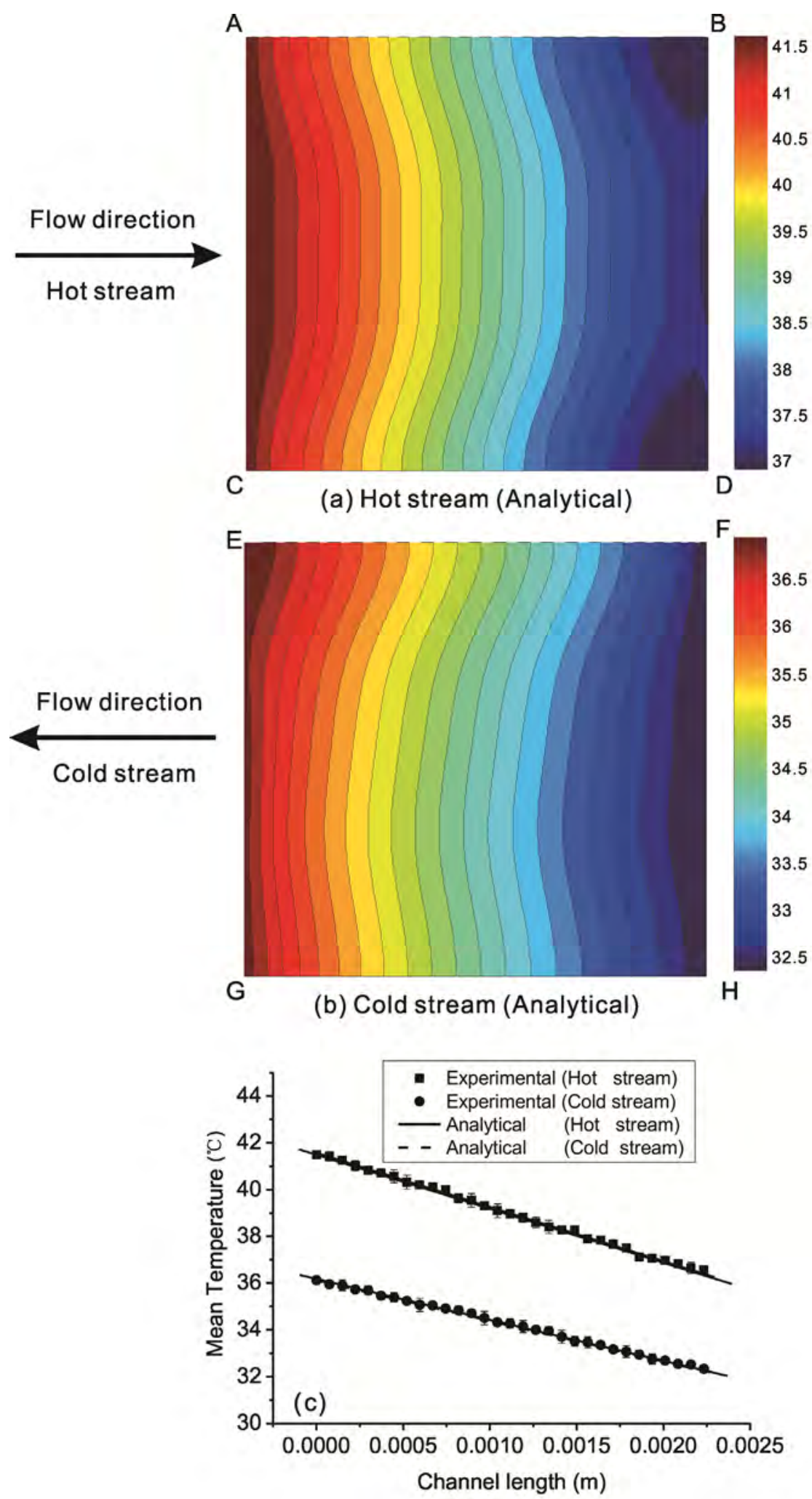

Figure 6. Comparison between analytical and experimental temperature distributions ( $\&=1000 \mu \mathrm{l} / \mathrm{h}$ ) : (a) Analytical temperature contours for hot stream, (b) analytical temperature contours for cold stream, (c) comparison of mean temperature profile for the hot and cold streams 